\title{
Combined Gas Electron Diffraction and Mass Spectrometric Experimental Setup at Bielefeld University
}

\author{
Yury V. Vishnevskiy ${ }^{* \dagger} \quad$ Sebastian Blomeyer ${ }^{\dagger} \quad$ Christian G. Reuter $^{\dagger}$ \\ Oleg A. Pimenov ${ }^{\ddagger} \quad$ Sergey A. Shlykov ${ }^{\ddagger}$
}

\section{Abstract}

We have designed and constructed a combined experimental setup for synchronous measurements of electron diffraction patterns and mass-spectra of gas samples. Test measurements have been performed for acetic acid at two temperatures, 296 and $457 \mathrm{~K}$, respectively. Electron diffraction data have been analysed taking into account mass spectra measured in the same experiments. From the diffraction intensities molecular structures and mole fractions of the acetic acid monomer and dimer have been refined. The obtained results demonstrate the importance of measuring mass spectra in gas electron diffraction experiments. In particular, it is possible to detect the sample decomposition, which can be used for the optimization of experimental conditions and for the data interpretation. The determined in this work length of the hydrogen bond in the acetic acid dimer, $r_{\mathrm{e}}(\mathrm{O} \cdots \mathrm{H})=1.657(9) \AA$, is in good agreement with modern theoretical predictions. We recommend to measure diffraction patterns of acetic acid for the calibration of the sample pressure in the diffraction point.

\footnotetext{
*Corresponding author, yury.vishnevskiy@uni-bielefeld.de

${ }^{\dagger}$ Lehrstuhl für Anorganische Chemie und Strukturchemie, Universität Bielefeld, Universitätsstraße 25, 33615 Bielefeld, Germany. Tel.: +495211066164 Fax: +495211066026

$\ddagger$ Ivanovo State University of Chemistry and Technology, Ivanovo, Russia
}

\section{Introduction}

Gas electron diffraction (GED) is one of the most important experimental methods for determination of molecular structure in the gas phase. In contrast to spectroscopic methods it has no intrinsic limitations connected with dipole moment and size of molecules [1]. On the other side GED has the disadvantage of measuring an integral signal. The intensity in each point of the diffraction pattern is a sum of contributions from all species in the diffraction volume. Thus, signals from different molecules are not separated in time and space but rather accumulated on a single detector. Generally the analysis of such data is an extremely ill-posed problem without additional information on the vapour composition. Due to this reason the majority of GED studies are done for pure and stable substances. However, there are many interesting compounds, which are thermally unstable or react under the experimental conditions. For example, a recent investigation of manganese(III) tris(acetylacetonate), an important example of the static Jahn-Teller effect, has been hampered by the thermal decomposition at the experimental conditions [2]. In this case additional information on vapour composition is crucial. In principle, mass spectrometry (MS) can be used for this purpose. The problem, however, is that in a standard MS setup it is hardly possible to simulate conditions of GED experiments accurately. The other problem is the impossibility to control GED measurements online. Only if attached directly to a GED unit and operated synchronously, a mass spectrometer can be used for the adjustment of experimental conditions. 
The first successful combination of a mass spectrometer with a gas electron diffractometer has been reported by Hargittai et al. [3]. This setup has been used for studying numerous compounds, for example aluminum trihalides [4]. The other combined instrument has been constructed by the Ivanovo group $[5,6]$. At present in the world this is the only experimental setup, which is routinely used for combined measurements of gas electron diffraction patterns and mass spectra. One of the latest results from this group is the molecular structure of manganese(II) bis-acetylacetonate, a very good example showing the importance of mass-spectrometric control in GED experiments [7].

At Bielefeld University we use a KD-G2 gas electron diffractometer, a heavily modified [8] version of the apparatus previously operated by Oberhammer at the University of Tübingen [9]. Recently a series of improvements for increasing operability and data quality have been applied [10]. In this contribution we describe for the first time our work on combining this GED unit with a quadrupole mass-spectrometer and show first results of test measurements.

\section{$3 \quad$ Experimental setup}

The scheme of the coupled gas electron diffractometer and mass-spectrometer units is provided in Figure 1. The photograph of the respective real setup at Bielefeld University is shown in Figure 2. The general principle for the combination of the two instruments was the reduction of their influence on each other. Most importantly, the electron beam in the diffraction chamber should not be deformed by any electromagnetic fields. A substance is introduced into the diffraction volume as a molecular jet, which has been clearly demonstrated in our previous work [10]. Most of the vapour is collected on surfaces of the cold trap and pumped out with the diffusion pump. A part of the jet goes through the first aperture and enters the chamber connected to the reservoir for liquid nitrogen. Here the molecular beam is formed and goes further through the other two apertures around the mechanical valve into the MS chamber. The valve has been designed and con- structed to be operated manually. Its independence from any electrical and air pressure connections ensures the absence of rapid pressure changes in the MS chamber. This is important in cases when the MS unit is detached for moving to another position (see flanges $\mathbf{L}, \mathbf{M}$ and $\mathbf{S}$ in Figure 1) and for maintenance of the GED unit. During adjustments of substance pressure in the diffraction chamber the valve is also closed in order to keep the vacuum in the MS unit as high as possible. In combined measurements the valve is opened, the molecular beam enters the MS chamber, analysed and pumped out with a water cooled turbo molecular pump Edwards EXT 75DX (pumping speed $61 \mathrm{l} \mathrm{s}^{-1}$ for $\mathrm{N}_{2}$ ). Thus, the differential pumping is used in the combined experimental setup to ensure the high vacuum in the MS chamber. An ion gauge Edwards AIGX-8 is used for measuring of pressure in the MS unit. The ion gauge and the turbo pump are operated with a control device Edwards TIC 100W. In closed state the typical pressure in the MS unit is about $1 \times 10^{-7} \mathrm{mbar}$. This value has been achieved using rubber ring sealings with vacuum grease. In measurements, when the valve is opened, the pressure raises up to $1-2 \times 10^{-6}$ mbar depending on the pressure in the diffraction chamber.

As quadrupole mass-spectrometer we use a commercial PIC 1000 series instrument from Hiden. Similar devices are used also in other mass-spectrometric investigations, see for example [11]. The instrument is equipped with two separate and interchangeable radio frequency generators. One is used for the extended mass range, up to $2500 \mathrm{amu}$. The other is for measurements with increased sensitivity for masses up to $510 \mathrm{amu}$. The probe is designed to be operated in high- and ultra-high vacuum conditions. The maximal operating pressure is determined by the SEM detector and is about $6 \times 10^{-6}$ mbar. Normally the mass-spectrometer is continuously scanning the required range of masses during the complete measurement session. The scanning time in the $1 \mathrm{amu}$ resolution mode is about 11 seconds per $100 \mathrm{amu}$.

The overall weight of the complete MS unit can achieve $50 \mathrm{~kg}$ depending on the configuration. Typically in GED experiments diffraction patterns are measured at least for two nozzle positions with different nozzle-to-detector distances. Thus the posi- 


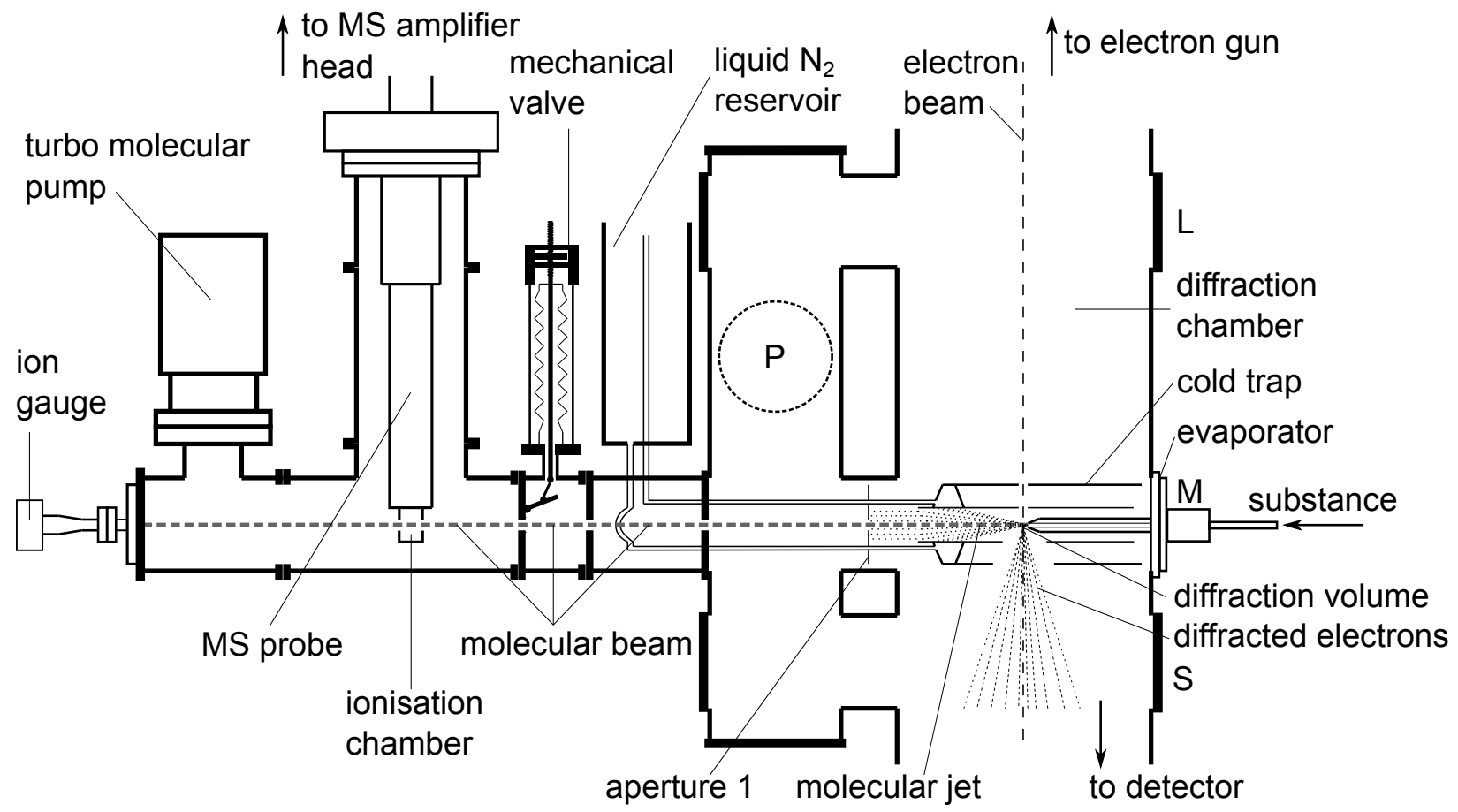

Figure 1: Scheme of the gas electron diffraction instrument combined with the quadrupole massspectrometer. $\mathbf{L}, \mathbf{M}$ and $\mathbf{S}$ indicate long, middle and short nozzle-to-detector camera settings, respectively. $\mathbf{P}$ shows connection to high vacuum diffusion pump on the back side of the chamber.

tion of the MS unit has to be frequently changed together with the nozzle. Taking into account the relatively large weight of the MS unit there have been constructed a special frame (see Figure 2) equipped with counterweight. This allows easy movement of the complete MS unit.

\section{Test measurements}

\subsection{Calibration}

In the background mode when the evaporator was closed the dominant signal in mass-spectra was at $m / z=18$, corresponding to water $\mathrm{H}_{2} \mathrm{O}^{+}$(see Figure 1 in Supporting Information). For the calibration of the mass-spectrometer perfluorotributylamine $\mathrm{N}\left(\mathrm{C}_{4} \mathrm{~F}_{9}\right)_{3}$ has been taken. This compound is an airstable volatile colorless liquid, well suitable for intro- duction of its vapor into the diffraction chamber without heating. The measured mass-spectra (see Figures 2 and 3 in SI) showed expected signals at $m / z=69$, 100, 113, 119, 131, 219, 264, 414 and $502 \mathrm{amu}$, which completely agrees with the data in NIST database [12]. Calibration of the electron diffractometer is described below.

\subsection{Acetic acid}

For testing of the combined experimental setup diffraction patterns and mass spectra of gaseous acetic acid (AA) have been measured synchronously. A commercial sample of AA (Busch-Chemie GmbH, technical grade, purity at least $99 \%$ ) has been used. Two series of measurements were performed, first without heating for $T=296 \mathrm{~K}$ and then with heating at $T=457 \mathrm{~K}$. For convenience, in this work we 


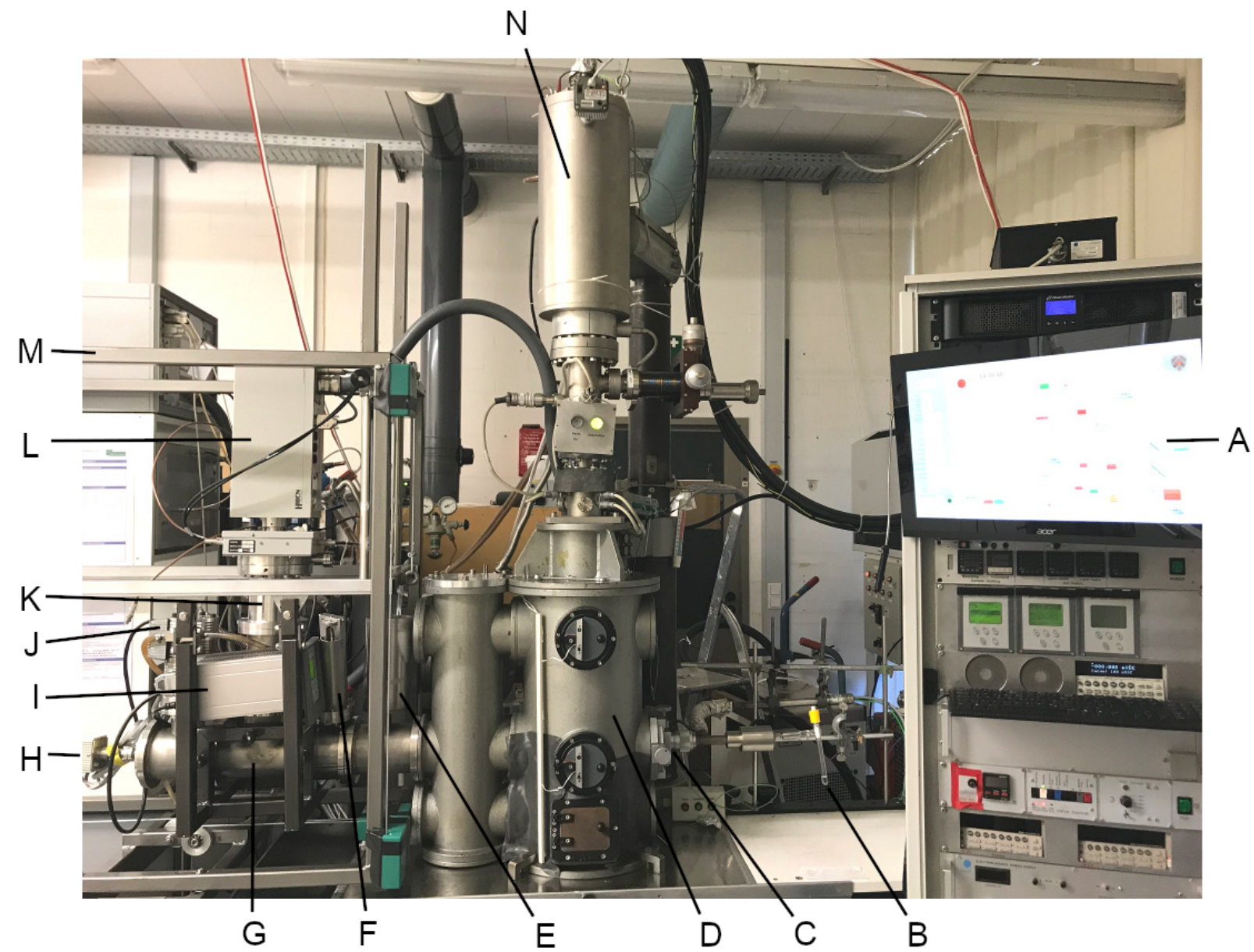

Figure 2: Photograph of the gas electron diffraction instrument at Bielefeld University combined with the quadrupole mass-spectrometer. A control panel, B ampule with substance, $\mathbf{C}$ evaporator (in the middle position on the photograph), $\mathbf{D}$ diffraction chamber, $\mathbf{E}$ cold trap reservoir for liquid nitrogen, $\mathbf{F}$ mechanical valve, $\mathbf{G}$ position of ionisation chamber, $\mathbf{H}$ ion gauge, $\mathbf{I}$ control device for turbo pump and ion gauge, $\mathbf{J}$ turbo molecular pump, $\mathbf{K}$ chamber with quadrupole mass-spectrometer probe, $\mathbf{L}$ amplifier head, $\mathbf{M}$ frame for the mass-spectrometer unit, $\mathbf{N}$ electron gun.

denote them as room-temperature (RT) and hightemperature (HT) experiments, although the latter can be accepted only in a relative sense. The conditions of the experiments are listed in Table 1. During the experiments mass spectra have been measured and recorded continuously, including time periods between exposures. Mass spectra in these periods corresponded to background. Figure 3 shows a typi- cal mass spectrum of AA measured during electron diffraction exposure for nozzle temperature $T=296 \mathrm{~K}$. Note, these data have been obtained by subtracting the background from the total spectrum. Thus the very small negative signals were due to experimental noise. Figure 4 shows mass spectrum of AA obtained in the same way for measurements at $T=457 \mathrm{~K}$.

Electron diffraction patterns were recorded on 
Table 1: Parameters of combined GED + MS experiments.

\begin{tabular}{|c|c|c|}
\hline Parameter & RT & HT \\
\hline$L_{\mathrm{nd}}{ }^{\mathrm{a}}, \mathrm{mm}$ & 250.0 & 250.0 \\
\hline$V_{\mathrm{ED}}{ }^{\mathrm{b}}, \mathrm{kV}$ & 60 & 60 \\
\hline$V_{\mathrm{MS}}{ }^{\mathrm{c}}, \mathrm{V}$ & 70 & 70 \\
\hline$I_{\mathrm{ED}}{ }^{\mathrm{d}}, \mu \mathrm{A}$ & 1.1 & 1.0 \\
\hline$I_{\mathrm{MS}}{ }^{\mathrm{e}}, \mu \mathrm{A}$ & 100 & 100 \\
\hline$T^{\mathrm{f}}, \mathrm{K}$ & $296(1)$ & $457(4)$ \\
\hline$P_{\mathrm{ED}}{ }^{\mathrm{g}}$, mbar & $2 \times 10^{-6}$ & $1 \times 10^{-6}$ \\
\hline$P_{\mathrm{MS}}{ }^{\mathrm{h}}, \mathrm{mbar}$ & $8 \times 10^{-7}$ & $9 \times 10^{-7}$ \\
\hline$t^{\mathrm{i}}, \mathrm{sec}$ & $8-12$ & 12 \\
\hline$s / \Delta s^{\mathrm{j}}, \AA^{-1}$ & $4.8-29.4 / 0.2$ & $4.8-29.4 / 0.2$ \\
\hline Plates $^{\mathrm{k}}$ sub/std & $4 / 1$ & $4 / 1$ \\
\hline Infl. points ${ }^{1}$ & $6 / 4$ & $6 / 4$ \\
\hline$\lambda^{\mathrm{m}}, \AA$ & 0.048797 & 0.048702 \\
\hline$w R_{\exp }{ }^{n}, \%$ & 4.81 & 7.21 \\
\hline
\end{tabular}

${ }^{\text {a }}$ Distance from nozzle tip to detector.

b Accelerating voltage for primary ED electron beam.

${ }^{c}$ Accelerating voltage for ionizing electrons in MS.

$\mathrm{d}$ Current in primary ED electron beam.

${ }^{\mathrm{e}}$ Emission of electrons in MS.

${ }^{\mathrm{f}}$ Temperature of nozzle tip.

${ }^{\mathrm{g}}$ Residual pressure in diffraction chamber during exposure.

${ }^{\mathrm{h}}$ Residual pressure in MS unit during exposure.

${ }^{\mathrm{i}}$ Exposure time.

j Covered and processed $s$-range, step size for $s$-values.

${ }^{\mathrm{k}}$ Number of successful exposures of sample and standard.

${ }^{1}$ Maximal allowed number of inflection points for background lines of sample and standard.

${ }^{m}$ Calibrated electron wavelength.

${ }^{\mathrm{n}}$ Weighted experimental $R$-factor [13] for $s M(s)$.

BAS-MP Imaging Plates. Gaseous $\mathrm{CCl}_{4}$ was used as a standard, for which diffraction patterns were also measured in the same experiments. For the purposes of this work only one camera setting has been used with middle nozzle position, as shown in Figure 1. The exposed plates were scanned using a calibrated
Fuji BAS-1800II reader. Data reduction of the digitalized patterns has been done according to our standard procedure [14]. Electron wavelengths were refined from the obtained intensity functions of $\mathrm{CCl}_{4}$ as usually [15] taking the most accurate available parameters [16].

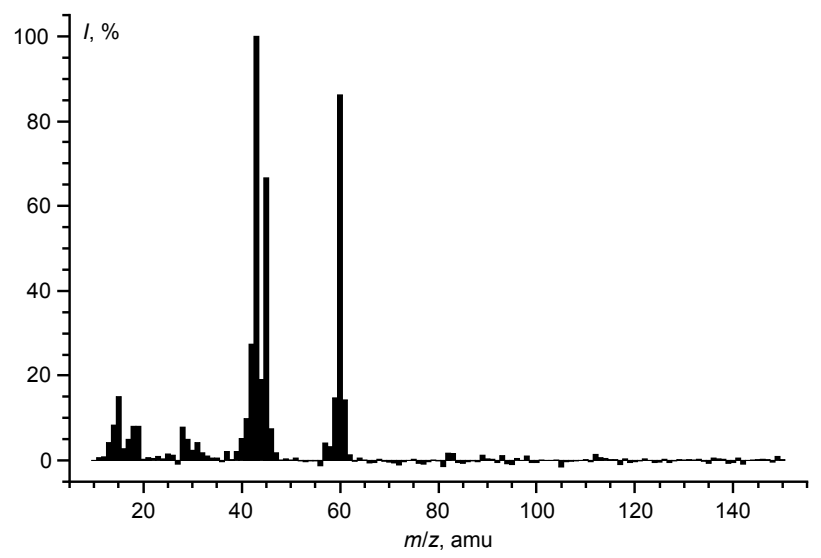

Figure 3: Mass spectrum of AA at $T=296 \mathrm{~K}$.

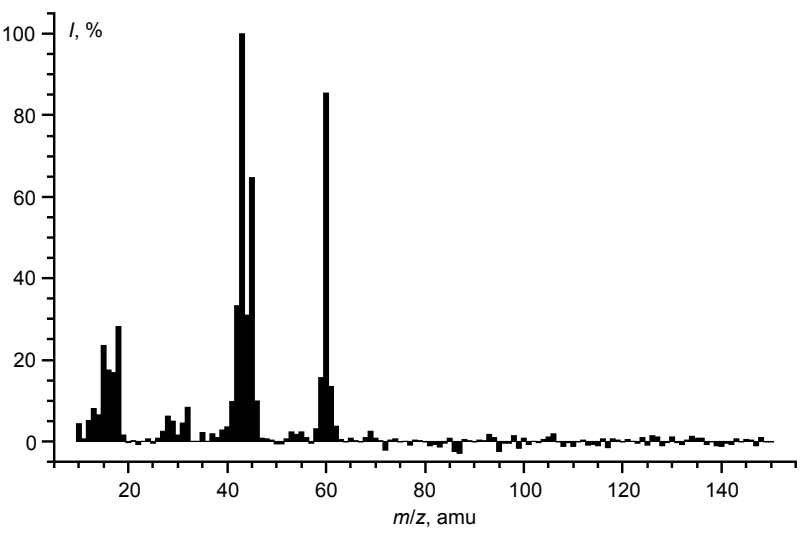

Figure 4: Mass spectrum of AA at $T=457 \mathrm{~K}$.

\section{Data analysis}

The measured mass spectra have been analysed and assigned. The most important signals are collected in Table 2. Experimental molecular intensity functions 
$s M(s)$ were obtained from the measured total intensity functions applying the multiplicative background procedure. Note, this was done using the reduced form of the total intensity as described in [16]. The procedure allows to control the line smoothness by defining the maximal number of inflection points for levelled background. For further processing averaged $s M(s)$ and their standard deviations were computed by combining individual $s M(s)$ from respective experiments.

Table 2: Relative intensities (\%) of selected signals in mass spectra of AA.

\begin{tabular}{lccc}
\hline Signal, amu & {$[12]$} & RT & HT \\
\hline $14, \mathrm{CH}_{2}{ }^{+}$ & 5 & 8 & 7 \\
$15, \mathrm{CH}_{3}{ }^{+}$ & 17 & 15 & 24 \\
$16, \mathrm{CH}_{4}{ }^{+}$ & 2 & 3 & 17 \\
$17, \mathrm{HO}^{+}$ & 1 & 5 & 17 \\
$18, \mathrm{H}_{2} \mathrm{O}^{+}$ & 3 & 8 & 28 \\
$28, \mathrm{CO}^{+}$ & 4 & 8 & 6 \\
$29, \mathrm{COH}^{+}$ & 8 & 5 & 5 \\
$42, \mathrm{H}_{2} \mathrm{CCO}^{+}$ & 13 & 27 & 33 \\
$43, \mathrm{H}_{3} \mathrm{CCO}^{+}$ & 100 & 100 & 100 \\
$44, \mathrm{CO}_{2}{ }^{+}$ & 2 & 19 & 31 \\
$45, \mathrm{COOH}^{+}$ & 90 & 67 & 65 \\
$60, \mathrm{H}_{3} \mathrm{CCOOH}^{+}$ & 75 & 86 & 85 \\
\hline
\end{tabular}

The analysis of the electron diffraction data has been carried out within the framework of the model of mixed AA monomer (see Figure 5) and dimer (Figure 6). Quantum-chemical calculations were performed to obtain supporting data required in the analysis of the electron diffraction intensities. Initial optimizations of molecular structures have been done using the ae-MP2/cc-pwCVTZ level of theory as implemented in the Gaussian package [17]. These structures were used as starting approximation in the refinement in UNEX program [18]. Additional optimizations of both, monomer and dimer, were done at the ae-CCSD $(\mathrm{T}) / \mathrm{cc}$-pwCVTZ level with the Cfour package [19]. The obtained theoretical structural parameters were utilized as flexible restraints in the regularizing method described earlier [20]. Next, structures and harmonic and cubic force fields were calculated at the B3LYP/cc-pVTZ level in Gaussian.
From these data interatomic vibrational amplitudes $l$ and corrections $\left(r_{\mathrm{e}}-r_{\mathrm{a}}\right)$ have been computed with the VibModule program [21]. For the mixture of AA monomer and dimer the reduced model molecular intensity functions $s M$ were calculated as

$$
s M=s \frac{x_{\mathrm{m}} I_{\mathrm{m}}^{\mathrm{mol}}+x_{\mathrm{d}} I_{\mathrm{d}}^{\mathrm{mol}}}{x_{\mathrm{m}} I_{\mathrm{m}}^{\text {at }}+x_{\mathrm{d}} I_{\mathrm{d}}^{\text {at }}}
$$

where $x_{\mathrm{m}}$ and $x_{\mathrm{d}}$ are mole fractions of the monomer and dimer, $I^{\mathrm{mol}}$ and $I^{\text {at }}$ are molecular and atomic scattering functions for the respective forms. Model $s M$ functions were fitted separately to the RT and HT experimental data, see Figure 7. The corresponding radial distribution functions are shown in Figures 8 and 9. Note, that the refinements of model parameters from both data sets were done in exactly the same manner, including the extent of regularization.

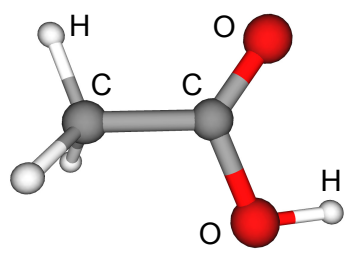

Figure 5: Molecular structure of the AA monomer.

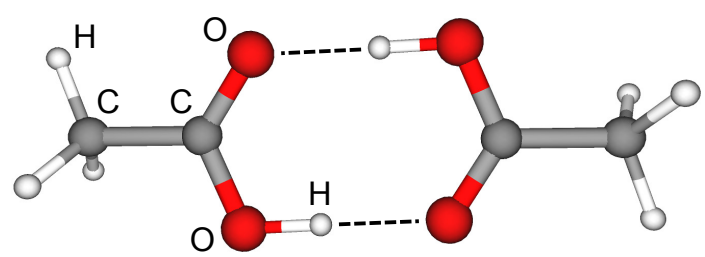

Figure 6: Molecular structure of the AA dimer. Dashed lines depict hydrogen bonds.

\section{Discussion}

The structure of the AA monomer and of its dimer has been studied using the GED method already in 1944 by Karle and Brockway [22]. The accuracy of their results was very limited and taking into account 


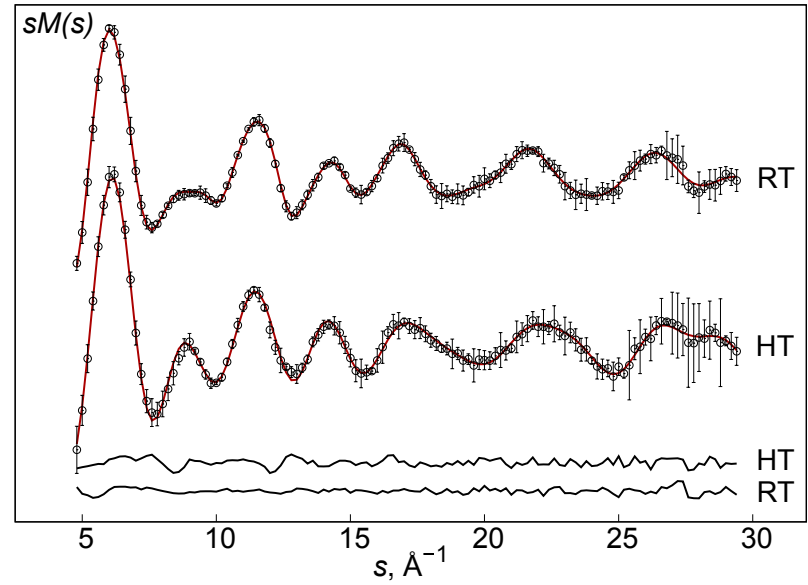

Figure 7: Experimental (dots) and model (lines) molecular intensity functions of AA in RT and HT experiments. Vertical bars are twofold standard deviations. Below are respective difference curves.

the importance of the substance another investigation has been performed in 1971 by Derissen [23]. However, in none of both studies the vapor composition were controlled. In case of thermal decomposition this could lead to an overinterpretation of experimental data resulting in shifted parameters. In this work the mass spectrometric measurements provided independent direct information on the composition of the sample in the diffraction chamber. The most important signals from mass spectra of AA are listed in Table 2. A comparison of the values from our RT experiment with those from NIST database demonstrated a general agreement for the most dominant signals at $m / z=43,45$ and 60 amu. However, the signals at $m / z=42,44$ were notably more intense in our spectra. This was probably related to the special conditions in our experimental setup and not to a decomposition of the substance. From the other side this was also supported by the fact that the respective electron diffraction data could be successfully interpreted assuming the existence of only AA monomer and dimer. Note, that none of the spectra contained signals of the dimer with $\mathrm{m} / \mathrm{z}>60$, although the analysis of the diffraction intensities unambiguously showed the presence of this form in RT experiment,

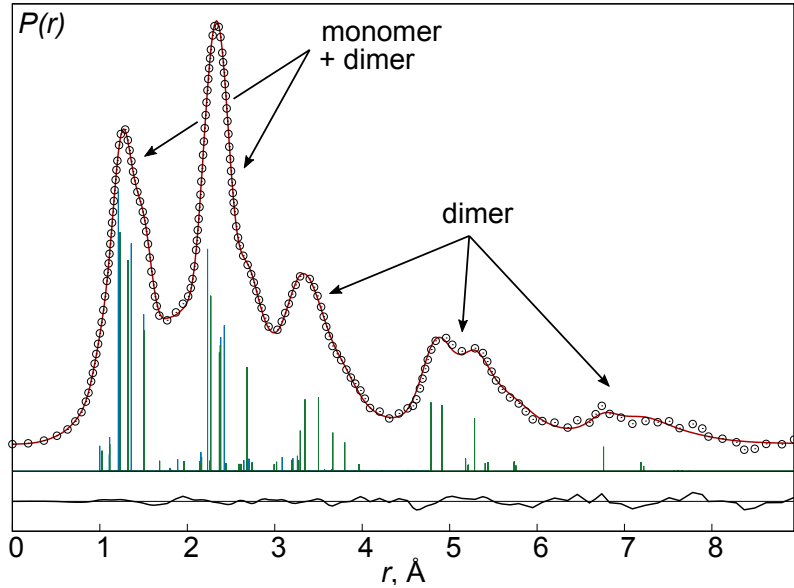

Figure 8: Experimental (dots) and model (line) radial distribution functions of AA in RT experiment. Vertical bars indicate contributions from interatomic distances.

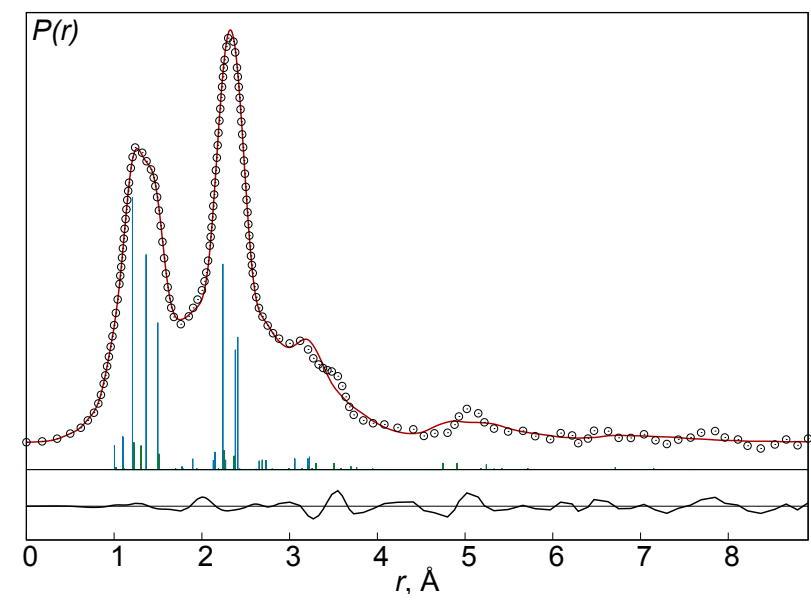

Figure 9: Experimental (dots) and model (line) radial distribution functions of AA in HT experiment. Vertical bars indicate contributions from interatomic distances.

which can also be clearly seen in the radial distribution function in Figure 8. This effect has been already observed earlier in a mass spectrometric study [24].

In the HT experiment the most intense signals in the mass spectra at $m / z=43,45$ and 60 amu re- 
Table 3: Selected theoretical and refined parameters of AA monomer and dimer. ${ }^{\mathrm{a}}$

\begin{tabular}{lccccc}
\hline Method & \multicolumn{2}{c}{ CCSD(T) } & \multicolumn{2}{c}{ GED RT } & GED HT \\
Parameter & monomer & dimer & monomer & dimer & monomer \\
\hline $\mathrm{C}-\mathrm{C}$ & 1.500 & 1.500 & $1.490(5)$ & $1.493(5)$ & $1.484(2)$ \\
$\mathrm{C}-\mathrm{O}$ & 1.354 & 1.319 & $1.350(3)$ & $1.315(4)$ & $1.354(2)$ \\
$\mathrm{C}=\mathrm{O}$ & 1.204 & 1.223 & $1.207(4)$ & $1.226(5)$ & $1.203(2)$ \\
$(\mathrm{C}-\mathrm{H})_{\mathrm{av}}$ & 1.087 & 1.087 & $1.090(7)$ & $1.090(7)$ & $1.076(8)$ \\
$\mathrm{O}-\mathrm{H}$ & 0.966 & 0.994 & $0.980(7)$ & $1.001(7)$ & $0.984(7)$ \\
$\mathrm{O} \ldots \mathrm{H}^{\mathrm{b}}$ & & 1.656 & & $1.657(9)$ & \\
$\mathrm{C}-\mathrm{C}-\mathrm{O}$ & 111.3 & 113.0 & $112.2(3)$ & $113.8(3)$ & $111.8(4)$ \\
$\mathrm{C}-\mathrm{C}=\mathrm{O}$ & 126.1 & 123.0 & $127.3(4)$ & $123.2(4)$ & $127.1(4)$ \\
$\mathrm{O}-\mathrm{C}=\mathrm{O}$ & 122.5 & 124.1 & $120.5(3)$ & $123.0(4)$ & $121.1(2)$ \\
$x^{\mathrm{c}}$ & & \multicolumn{5}{c}{$69(2)$} & $31(2)$ & $95(2)$ \\
$w R^{\mathrm{d}}$ & & \multicolumn{3}{c}{4.72} & 10.37 \\
\hline
\end{tabular}

a Bond lengths $(\AA)$ and angles (degrees) correspond to equilibrium structure. Least-squares standard deviations are in parentheses.

${ }^{\mathrm{b}}$ Hydrogen bonds, depicted as dashed lines in Figure 6 .

${ }^{\mathrm{c}}$ Refined mole fraction (\%) of the respective form.

${ }^{\mathrm{d}}$ Weighted factor of disagreement (\%) between model and experimental $s M(s)$.

mained unchanged in comparison to RT measurements. In contrast, the signals at $m / z=15,16,17,18$ and 44 increased significantly. The peak at $m / z=42$ was also larger. Earlier investigations [25, 26, 27] revealed two major channels of AA thermal decomposition, (a) the decarboxylation path resulting in methane and carbon dioxide and (b) decomposition to ketene and water. Thus the increased signals can be explained by contributions from molecular and fragment ions of the respective products of decomposition, namely $\mathrm{CH}_{3}{ }^{+}, \mathrm{CH}_{4}{ }^{+}, \mathrm{HO}^{+}, \mathrm{H}_{2} \mathrm{O}^{+}, \mathrm{H}_{2} \mathrm{CCO}^{+}$ and $\mathrm{CO}_{2}{ }^{+}$. The partial decomposition of AA can explain the significantly worse fit of the HT electron diffraction data in comparison to the RT data; this can be seen in the difference curves for $s M(s)$ (Figure 7) and for radial distribution functions (compare Figures 8 and 9). Although in the HT experiment the temperature was not very high $(457 \mathrm{~K})$, the decomposition could be stimulated by brass surfaces along the rather long channel of the used evaporator [8]. Earlier even in an all-quartz apparatus for viscosity measurements thermal decomposition of AA was detected at about $480 \mathrm{~K}$ and low densities of the substance [28], which resembles our experimental conditions.

Parameters of AA determined from both data sets and the corresponding best theoretical values are collected in Table 3. First we discuss the values of parameters refined from RT data. The major effect of the dimerization on the geometrical parameters predicted by theory is the shortening of the single $\mathrm{C}-\mathrm{O}$ bonds and the elongation of the $\mathrm{C}=\mathrm{O}$ double bonds. The refined values proved this prediction; this was also seen in the previous investigation [23]. For the $\mathrm{C}-\mathrm{C}$ bonds the theoretical values were essentially the same in the monomer and dimer. The respective refined values did not differ within the stated standard deviations but were systematically lower. In contrast, the values from the work of Derissen are significantly different, $r_{\mathrm{a}}=1.520(5)$ and 1.506(5) $\AA$ for monomer and dimer, respectively. Note, that all parameters in our work have been refined independently and without any rigid constraints. Taking into account the relatively high general accuracy of the coupled cluster theory for molecules consisting of atoms of the first and second periods (see for example [29] and references therein), these contradictions between theory 
and experiment should be studied in more detail in the future. Another notable result of our study was the refined length of the hydrogen bonds $r_{\mathrm{a}}(\mathrm{O} \cdots \mathrm{H})$ $=1.672(9) \AA$. This value is in good agreement with the modern theoretical prediction, but deviates significantly from the previous experimental value $r_{\mathrm{a}}=$ $2.680(10) \AA[23]$.

One of the most interesting refined parameters was the ratio of the monomeric and dimeric forms of AA in experiments at different temperatures. Since the mass spectra did not show any signals specific for the dimer, the only source of experimental data for its detection were the electron diffraction intensities. Even without refinement a visual inspection of the experimental molecular intensity functions showed the differences between RT and HT data (see Figure 7). In the radial distribution functions (Figures 8 and 9) they could be seen much clearer starting from $r=3$ $\AA$ due to contributions of long interatomic distances in the dimer. The least-squares refinements resulted in mole fractions for monomer of $69(2) \%$ and $95(2)$ $\%$ in RT and HT experiments, respectively. It should be noted that we used strict criteria for background lines and tested different refinement strategies. In all cases refinements of the composition parameter $x$ were stable and independent on its initial approximation. However, the calculation of total uncertainties including systematic errors was beyond the scope of this work, thus the obtained values should be taken with care. Still, we can note a great deviation of the refined $x=69(2)$ mole $\%$ for monomer at $T=296(1)$ $\mathrm{K}$ in this work from the published data of Derissen of $13.5(30) \%$ for the nearly the same temperature $T=$ $297(3)$ K. A reason for that could be the very large drop of the vapor pressure within the inlet system due to the aforementioned long channel of the used evaporator. In addition, during the measurements the valve in the evaporator was opened only slightly, which also reduced the pressure in the diffraction volume. In Table S1 of the Supplementary Information we have collected composition parameters of AA calculated for different conditions from the reported earlier thermodynamic data [30]. Comparing these values with the refined $x$ parameters it can be concluded that the pressure of AA vapor at the diffraction point of the RT experiment was around 0.1-1.0 mbar, which is a realistic range for this setup. Concerning the HT experiment, we obtained, expectedly, a larger contribution of monomer but not exactly 100 $\%$ due to decomposition. The products of decomposition have not been included into the model and the total weighted $R$-factor was relatively large, $10.4 \%$. On the other side, the experimental $R$-factor, showing the reproducibility of the experimental HT data, was also unusually large, $7.2 \%$. This could be due to the instability of the thermal decomposition processes. Thus, we intentionally rejected improving the model and increasing its complexity to avoid overinterpretation of the experimental data. Some of the refined from HT data parameters were probably affected by the described problems. For example, the averaged length of the $\mathrm{C}-\mathrm{H}$ bonds was lower possibly due to unaccounted contributions from water $\mathrm{H}_{2} \mathrm{O}$ and ketene $\mathrm{H}_{2} \mathrm{C}=\mathrm{C}=\mathrm{O}$.

In general, a thorough investigation of the structure of AA was not the goal of the present study. For obtaining more accurate and precise parameters additional measurements are required, first of all for the long nozzle-to-detector camera setting in order to get small angle scattering data. Rotational constants, if any, can also be taken into account. Finally, after calculation of total errors of experimental parameters, a well grounded discussion for the molecular structure of AA can be conducted.

\section{Conclusions and outlook}

The main purpose of this paper was the description of the experimental setup for combined mass spectrometric and gas electron diffraction measurements at Bielefeld University. The test measurements of acetic acid demonstrated the importance of such experiments under the special conditions of the GED unit. The partial thermal decomposition observed in mass spectra prevented overfitting of the model to the experimental electron diffraction intensities recorded at elevated temperatures. Taking into account the results of this study a more complete and accurate investigation of acetic acid can be performed by improving the evaporator for preventing the thermal decomposition and by measuring diffraction data for a 
wider range of scattering angles. We can also conclude that acetic acid can probably be used as a standard substance for calibration of sample pressure in diffraction point of GED experiments.

\section{Acknowledgments}

This work was supported by Deutsche Forschungsgemeinschaft (DFG, grant VI 713/1-2). Many thanks to the mechanical workshop of the chemical faculty at the Bielefeld University, especially to Manfred Hoffmann and Jan-Ulrich Klamert. YuVV is grateful to HPC facilities at the Universität zu Köln for providing computational time and programs. Special thanks to Norbert W. Mitzel for fruitful discussions and support.

\section{Compliance with ethical stan- dards}

The authors declare no competing financial interest.

\section{Keywords}

gas electron diffraction, mass spectrometry, molecular structure, acetic acid

\section{References}

[1] R. A. Bonham and M. Fink. High-energy electron scattering. Van Nostrand Reinhold, New York, 1974.

[2] R. J. F. Berger, G. V. Girichev, N. I. Giricheva, A. A. Petrova, and N. V. Tverdova. The Structure of $\mathrm{Mn}(\mathrm{acac})_{3}$ - Experimental Evidence of a Static Jahn-Teller Effect in the Gas Phase. Angew. Chem. Int. Ed., 56(49):15751-15754, 2017.

[3] I. Hargittai, S. Bohatka, J. Tremmel, and I. Berecz. Gas Electron Diffraction Part III. Experiments Combined with Quadrupole Mass
Spectromentry. Hung. Sci. Instrum., 50:51-56, 1980.

[4] Z. Varga, M. Kolonits, and M. Hargittai. Comprehensive study of the structure of aluminum trihalides from electron diffraction and computation. Struct. Chem., 23(3):879-893, 2012.

[5] Georgiy V. Girichev, Sergey A. Shlykov, and $\mathrm{Yu}$. F. Revichev. Apparatus for study of molecular structure of valence-unsaturated compounds (in Russian). Prib. Tekh. Eksp., 29(4):167-169, 1986.

[6] N. I. Giricheva, G. V. Girichev, S. A. Shlykov, V. A. Titov, and T. P. Chusova. The joint gas electron diffraction and mass spectrometric study of $\mathrm{GeI}_{4}(\mathrm{~g})+\mathrm{Ge}(\mathrm{s})$ system. Molecular structure of germanium diiodide. J. Mol. Struct., 344(1):127-134, 1995.

[7] R. J. F. Berger, G. V. Girichev, N. I. Giricheva, A. A. Otlyotov, and A. A. Petrova. Ligand Coordination in Bis( $\beta$-diketonato) d Metals: The $\mathrm{Mn}(\mathrm{II})$ Case of $D_{2 h}$ versus $D_{2 d}$ Symmetry. Inorg. Chem., 58(7):4344-4349, 2019.

[8] R. J. F. Berger, M. Hoffmann, S. A. Hayes, and N. W. Mitzel. An Improved Gas Electron Diffractometer - The Instrument, Data Collection, Reduction and Structure Refinement Procedures. Z. Naturforsch. B, 64b(11/12):1259 1268, 2009.

[9] H. Oberhammer. Developments in the apparatus used in Europe, including U.S.S.R., for studies of electron diffraction in vapours. In G. A. Sim and L. E. Sutton, editors, Molecular Structure by Diffraction Methods, volume 4, pages 24-44. The Chemical Society, Burlington House, London, 1976.

[10] C. G. Reuter, Y. V. Vishnevskiy, S. Blomeyer, and N. W. Mitzel. Gas electron diffraction of increased performance through optimization of nozzle, system design and digital control. $Z$. Naturforsch. B, 71(1):1-13, 2016. 
[11] W. A. D. Pires, K. L. Nixon, S. Ghosh, R. F. C. Neves, H. V. Duque, R. A. A. Amorim, D. B. Jones, F. Blanco, G. Garcia, M. J. Brunger, and M. C. A. Lopes. Electron impact ionization of 1-propanol. Int. J. Mass Spectrom., 422:32-41, 2017.

[12] W. E. Wallace. Mass Spectra. In P. J. Linstrom and W. G. Mallard, editors, NIST Chemistry WebBook, NIST Standard Reference Database Number 69. National Institute of Standards and Technology, Gaithersburg MD, 20899.

[13] Yu. V. Vishnevskii, I. F. Shishkov, L. V. Khristenko, A. N. Rykov, L. V. Vilkov, and H. Oberhammer. Molecular Structures of $o$ - and $m$-Fluoro(trifluoromethoxy)benzenes According to Gas Electron Diffraction and QuantumChemical Studies: Comparison of the Structures of Trifluoromethoxybenzene and Its Fluorinated Derivatives. Russ. J. Phys. Chem., 79(10):1537$1547,2005$.

[14] Yu. V. Vishnevskiy. The Initial Processing of the Gas Electron Diffraction Data: an Improved Method for Obtaining Intensity Curves from Diffraction Patterns. J. Mol. Struct., 833:30-41, 2007.

[15] Yu. V. Vishnevskiy. The Initial Processing of the Gas Electron Diffraction Data: New Method for Simultaneous Determination of the Sector Function and Electron Wavelength from Gas Standard Data. J. Mol. Struct., 871(1-3):24-32, 2007.

[16] Yury V. Vishnevskiy, Sebastian Blomeyer, and Christian G. Reuter. Gas standards in gas electron diffraction: accurate molecular structures of $\mathrm{CO}_{2}$ and $\mathrm{CCl}_{4}$. Struct. Chem., 31(2):667-677, 2020 .

[17] M. J. Frisch, G. W. Trucks, H. B. Schlegel, G. E. Scuseria, M. A. Robb, J. R. Cheeseman, G. Scalmani, V. Barone, G. A. Petersson, H. Nakatsuji, X. Li, M. Caricato, A. V. Marenich, J. Bloino, B. G. Janesko, R. Gomperts, B. Mennucci, H. P. Hratchian, J. V. Ortiz, A. F. Izmaylov, J. L. Sonnenberg, D. Williams-Young, F. Ding, F. Lipparini, F. Egidi, J. Goings, B. Peng, A. Petrone,
T. Henderson, D. Ranasinghe, V. G. Zakrzewski, J. Gao, N. Rega, G. Zheng, W. Liang, M. Hada, M. Ehara, K. Toyota, R. Fukuda, J. Hasegawa, M. Ishida, T. Nakajima, Y. Honda, O. Kitao, H. Nakai, T. Vreven, K. Throssell, J. A. Montgomery, Jr., J. E. Peralta, F. Ogliaro, M. J. Bearpark, J. J. Heyd, E. N. Brothers, K. N. Kudin, V. N. Staroverov, T. A. Keith, R. Kobayashi, J. Normand, K. Raghavachari, A. P. Rendell, J. C. Burant, S. S. Iyengar, J. Tomasi, M. Cossi, J. M. Millam, M. Klene, C. Adamo, R. Cammi, J. W. Ochterski, R. L. Martin, K. Morokuma, O. Farkas, J. B. Foresman, and D. J. Fox. Gaussian 16 Revision B.01, 2016. Gaussian Inc. Wallingford CT.

[18] Yu. V. Vishnevskiy, 2020, UNEX version 1.6, http://unexprog.org (accessed Mon Jan 20 2020).

[19] J. F. Stanton, J. Gauss, L. Cheng, M. E. Harding, D. A. Matthews, and P. G. Szalay. CFOUR, Coupled-Cluster techniques for Computational Chemistry, a quantum-chemical program package. With contributions from A.A. Auer, R.J. Bartlett, U. Benedikt, C. Berger, D.E. Bernholdt, Y.J. Bomble, O. Christiansen, F. Engel, R. Faber, M. Heckert, O. Heun, M. Hilgenberg, C. Huber, T.-C. Jagau, D. Jonsson, J. Jusélius, T. Kirsch, K. Klein, W.J. Lauderdale, F. Lipparini, T. Metzroth, L.A. Mück, D.P. O'Neill, D.R. Price, E. Prochnow, C. Puzzarini, K. Ruud, F. Schiffmann, W. Schwalbach, C. Simmons, S. Stopkowicz, A. Tajti, J. Vázquez, F. Wang, J.D. Watts and the integral packages MOLECULE (J. Almlöf and P.R. Taylor), PROPS (P.R. Taylor), ABACUS (T. Helgaker, H.J. Aa. Jensen, P. Jørgensen, and J. Olsen), and ECP routines by A. V. Mitin and C. van Wüllen. For the current version, see http://www.cfour.de.

[20] Yu. V. Vishnevskiy, M. A. Abaev, A. N. Rykov, M. E. Gurskii, P. A. Belyakov, S. Yu. Erdyakov, Yu. N. Bubnov, and N. W. Mitzel. Structure and bonding nature of the strained lewis acid 3-methyl-1-boraadamantane: A case study employing a new data-analysis procedure 
in gas electron diffraction. Chem. Eur. J., 18(34):10585-10594, 2012.

[21] Yu. V. Vishnevskiy and Yu. A. Zhabanov. New implementation of the first-order perturbation theory for calculation of interatomic vibrational amplitudes and corrections in gas electron diffraction. J. Phys.: Conf. Ser., 633(1):012076, 2015 .

[22] J. Karle and L. O. Brockway. An Electron Diffraction Investigation of the Monomers and Dimers of Formic, Acetic and Trifluoroacetic Acids and the Dimer of Deuterium Acetate. $J$. Am. Chem. Soc., 66(4):574-584, 1944.

[23] J. L. Derissen. A reinvestigation of the molecular structure of acetic acid monomer and dimer by gas electron diffraction. J. Mol. Struct., 7(1):6780, 1971.

[24] K. D. Cook and J. W. Taylor. A mass spectrometric study of the effect of supersonic molecular beam sampling on the clustering of acetic, acid vapor. Int. J. Mass Spectrom. Ion Phys., 35(3):259-271, 1980.

[25] P. G. Blake and G. E. Jackson. The thermal decomposition of acetic acid. J. Chem. Soc. B, pages $1153-1155,1968$.

[26] J. C. Mackie and K. R. Doolan. Hightemperature kinetics of thermal decomposition of acetic acid and its products. Int. J. Chem. Kinet., 16(5):525-541, 1984.

[27] M. T. Nguyen, D. Sengupta, G. Raspoet, and L. G. Vanquickenborne. Theoretical Study of the Thermal Decomposition of Acetic Acid: Decarboxylation Versus Dehydration. J. Phys. Chem., 99(31):11883-11888, 1995.

[28] E. Vogel and E. Bich. Dimerization and Viscosity of Acetic Acid Vapor. Z. Phys. Chem., 227:315-332, 2013.

[29] N. Vogt, I. I. Marochkin, and A. N. Rykov. Experiment and theory at the convergence limit: accurate equilibrium structure of picolinic acid by gas-phase electron diffraction and coupledcluster computations. Phys. Chem. Chem. Phys., 20(15):9787-9795, 2018.

[30] W. Weltner. The Vibrational Spectrum, Associative and Thermodynamic Properties of Acetic Acid Vapor. J. Am. Chem. Soc., 77(15):3941$3950,1955$. 
Combined Gas Electron Diffraction and Mass Spectrometric Experimental Setup at Bielefeld University

\section{Supporting Information}

Yury V. Vishnevskiy, Sebastian Blomeyer, Christian G. Reuter,

Oleg A. Pimenov, Sergey A. Shlykov

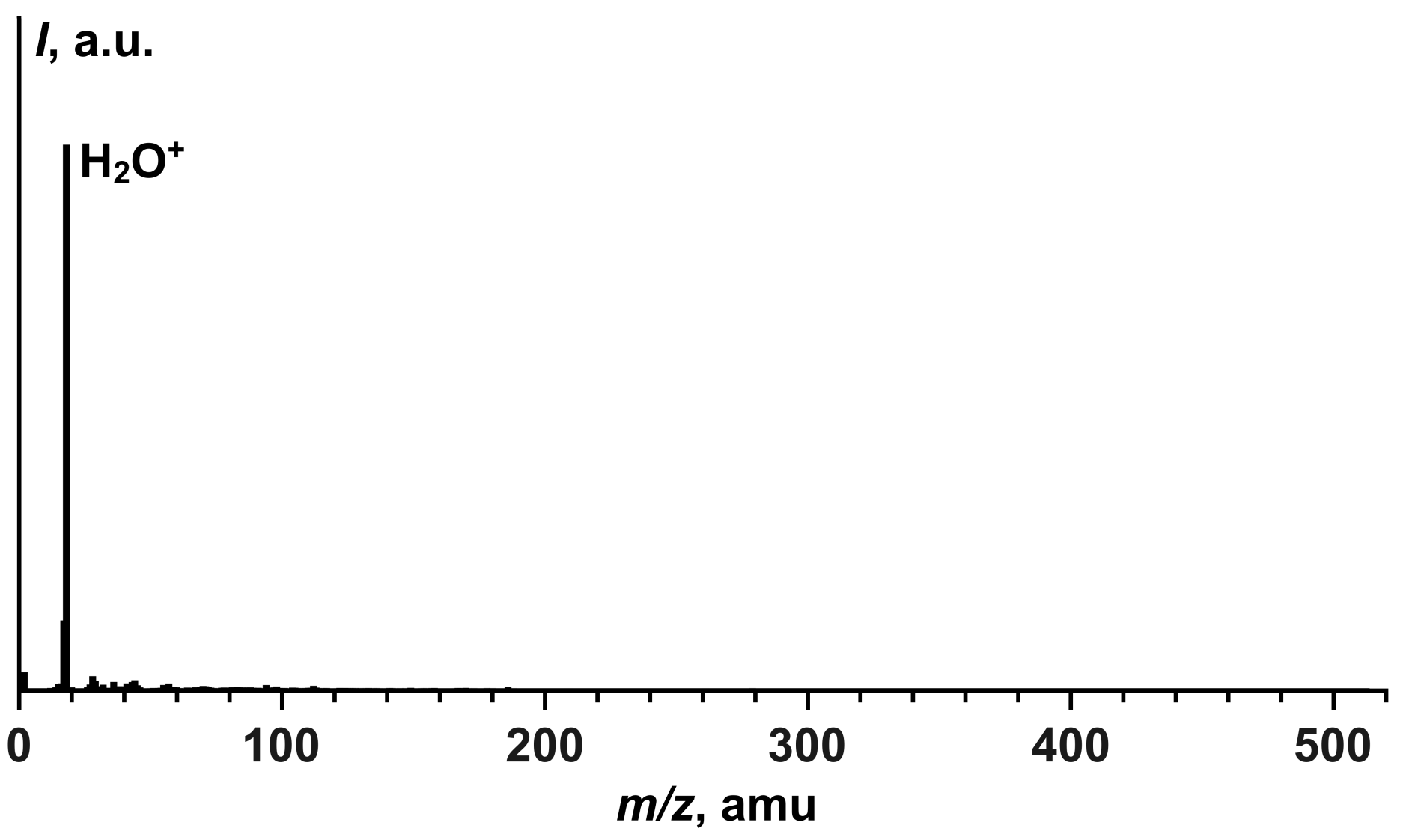

Figure S1: Background mass spectrum. 


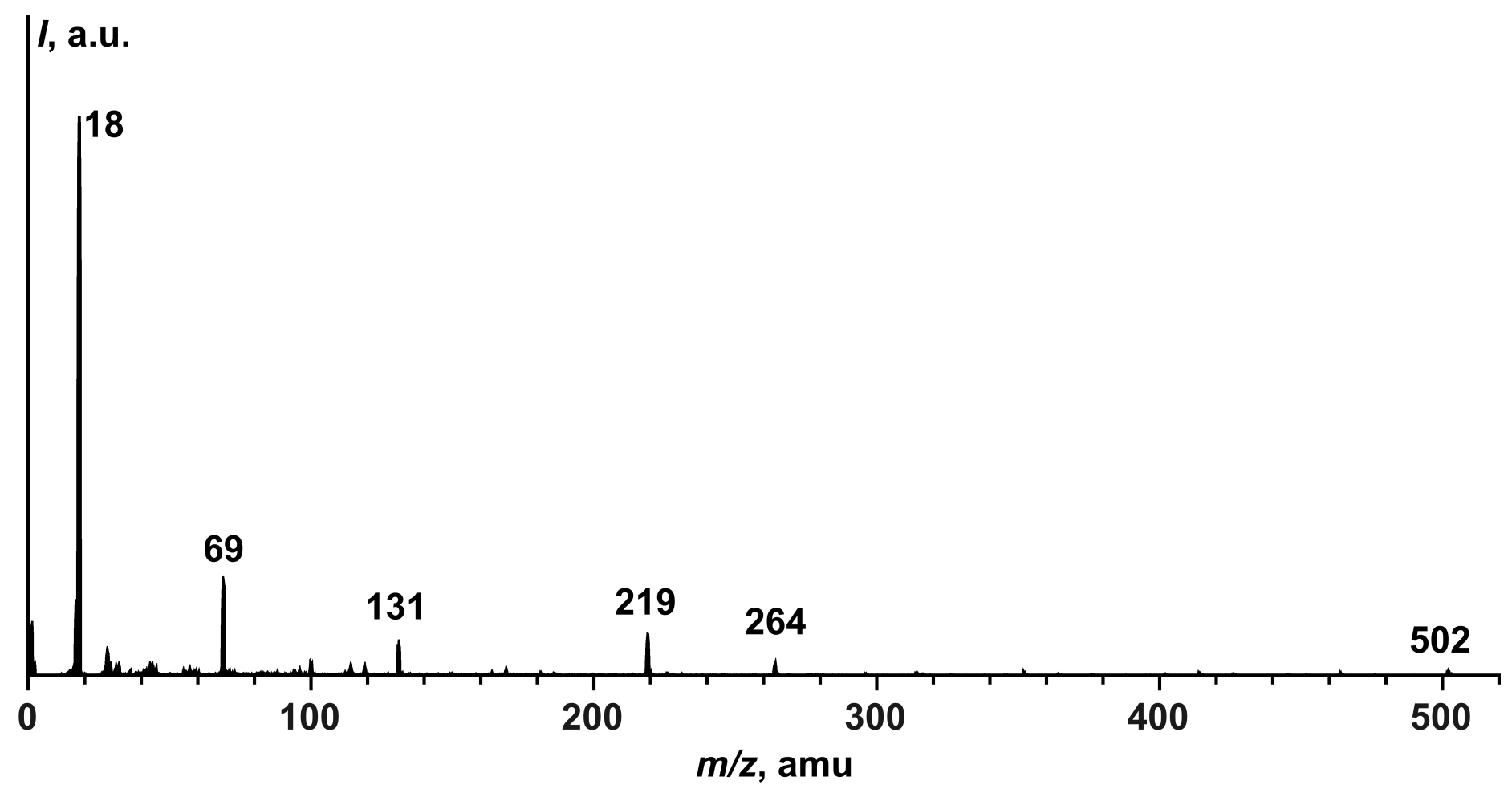

Figure S2: Mass spectrum of perfluorotributylamine with background water.

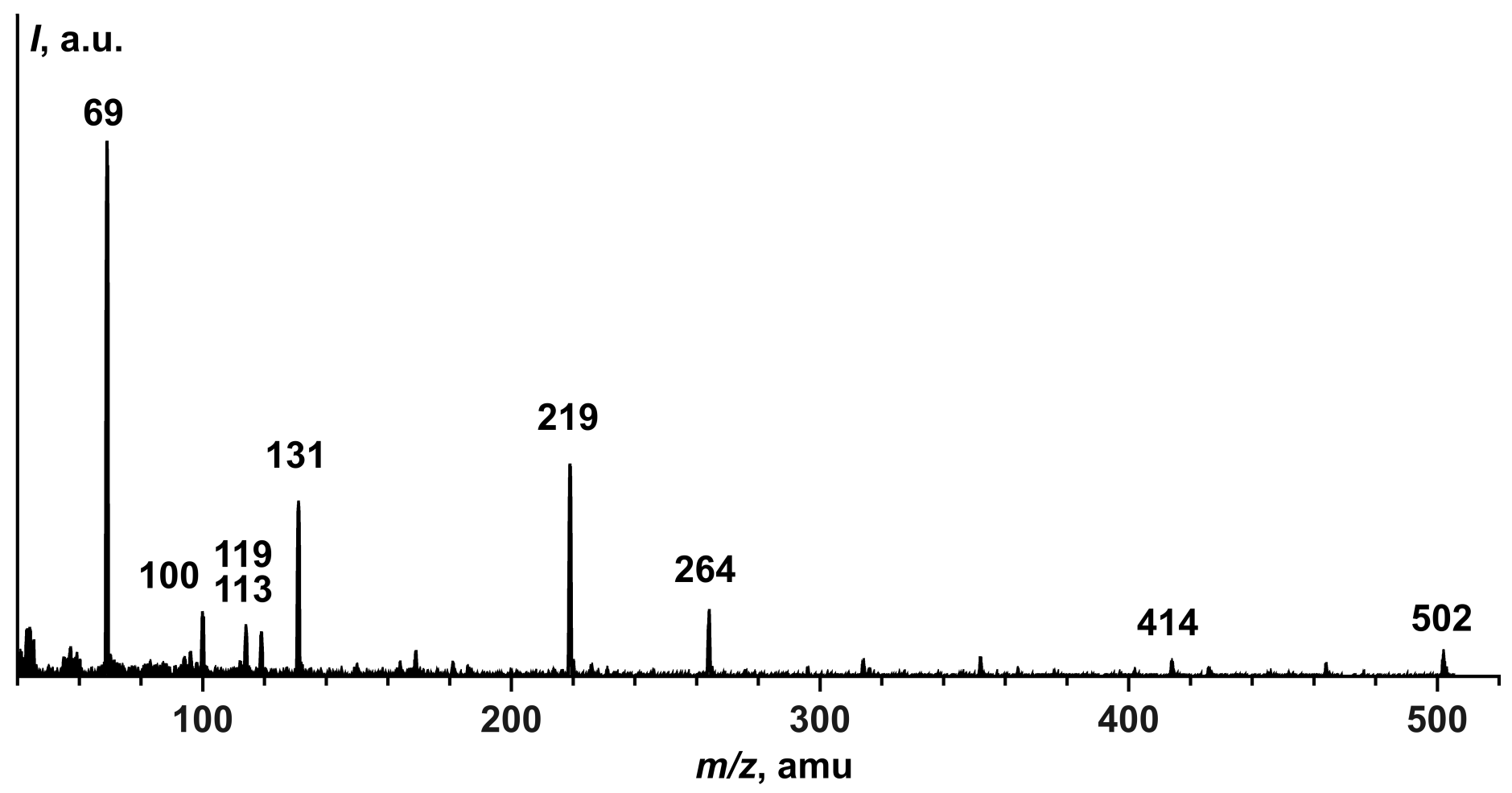

Figure S3: Mass spectrum of perfluorotributylamine. Signal of background water excluded for clarity. 


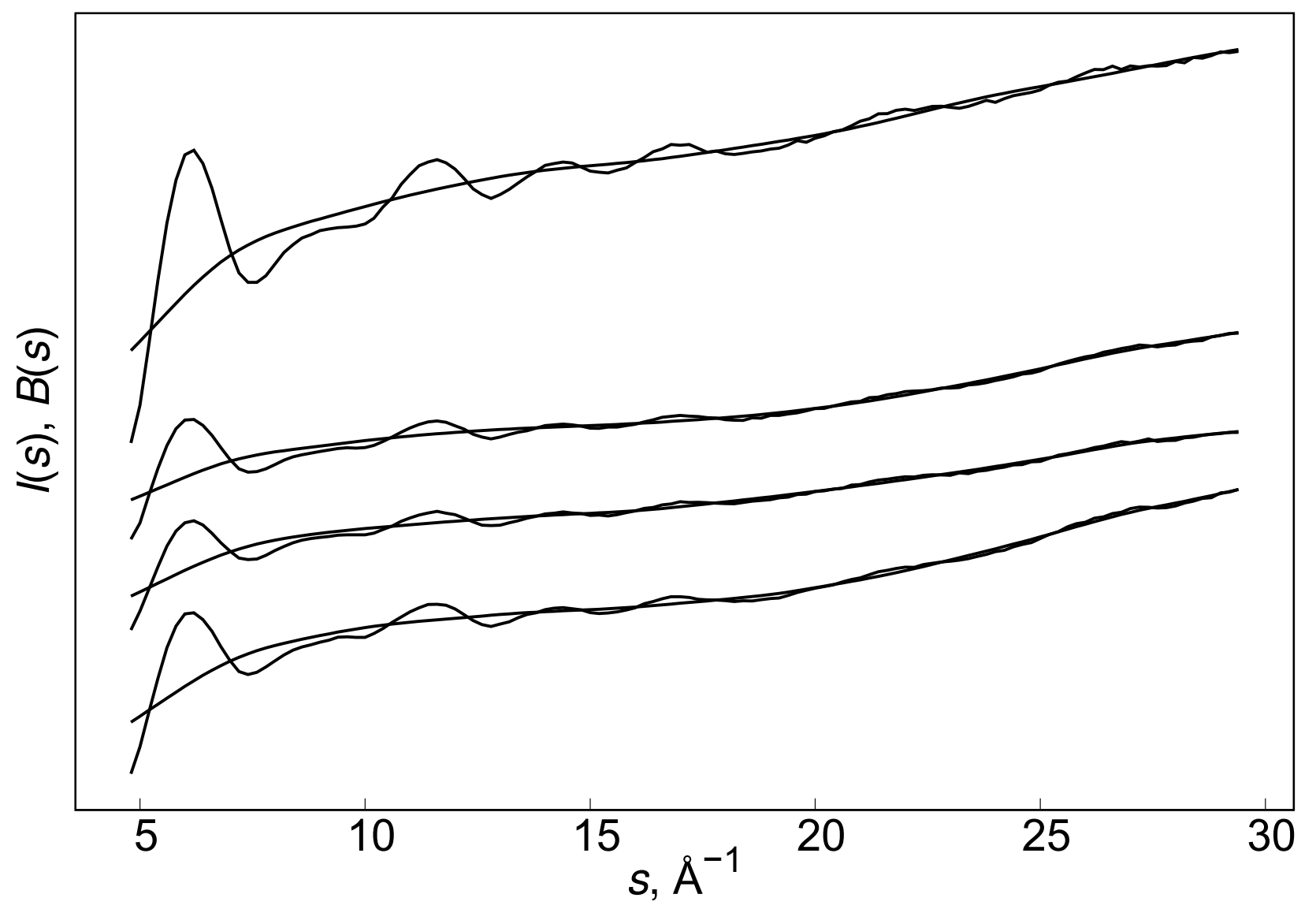

Figure S4: Reduced electron diffraction intensities and background lines of AA in RT experiment.

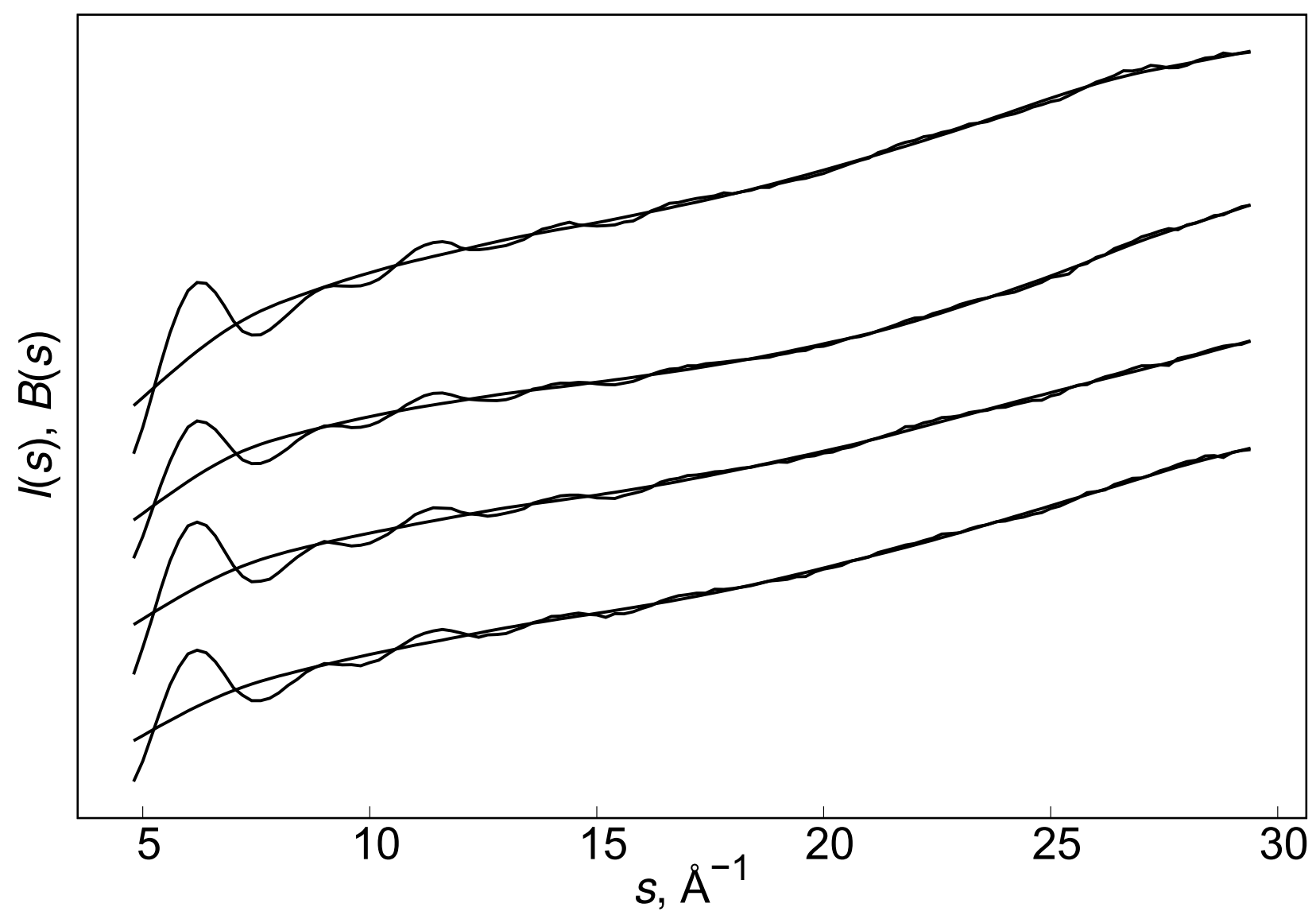

Figure S5: Reduced electron diffraction intensities and background lines of AA in HT experiment. 


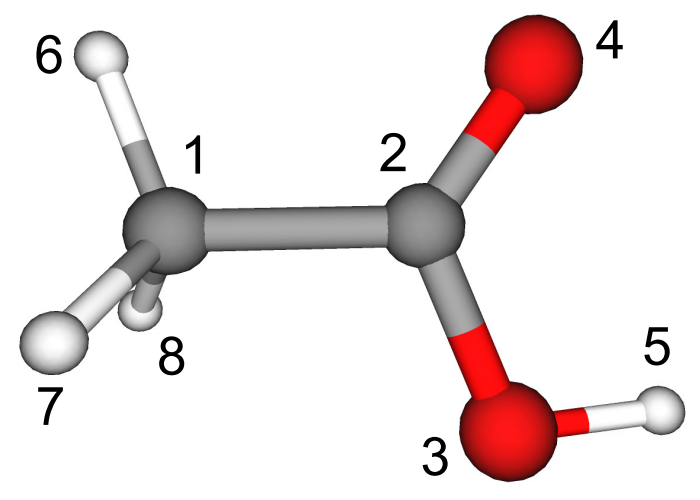

Figure S6: Internal numeration of atoms in AA monomer.

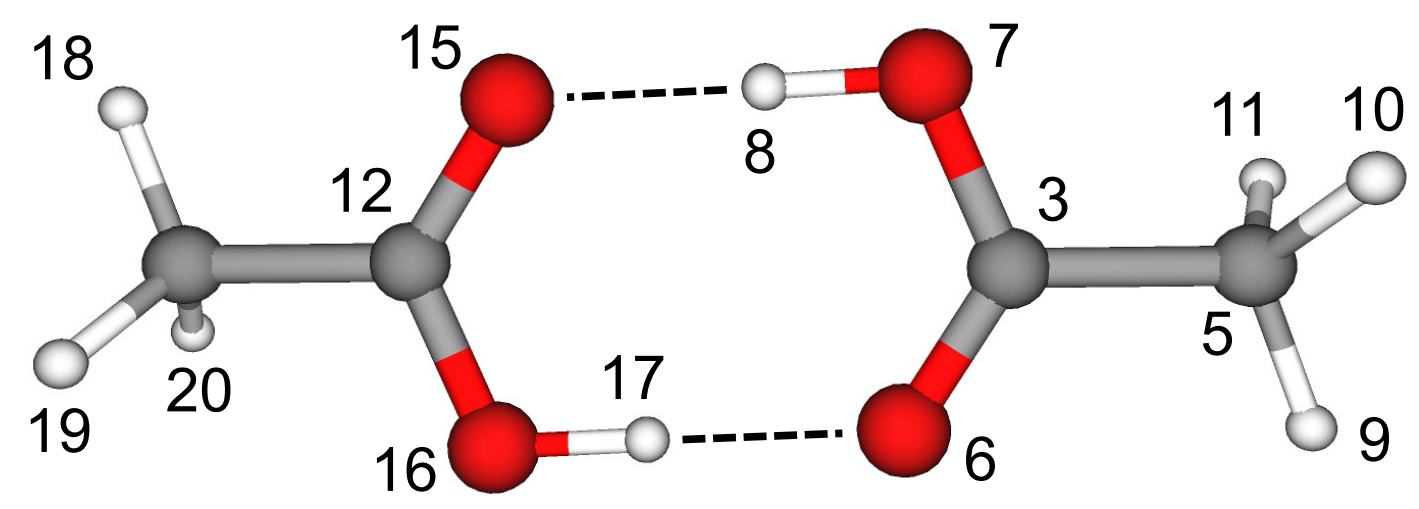

Figure S7: Internal numeration of atoms in AA dimer. 
Table S1: Composition ${ }^{\mathrm{a}}$ of AA vapor in different conditions

\begin{tabular}{rrrrr}
\hline$P$, mbar & $n_{\mathrm{m}}$ & $n_{\mathrm{d}}$ & $x_{\mathrm{m}}$ & $x_{\mathrm{d}}$ \\
\hline \multicolumn{5}{c}{$T=296 \mathrm{~K}$} \\
0.1 & 0.7848 & 0.1076 & 87.94 & 12.06 \\
1.0 & 0.3717 & 0.3142 & 54.19 & 45.81 \\
10.0 & 0.1256 & 0.4372 & 22.32 & 77.68 \\
100.0 & 0.0400 & 0.4800 & 7.69 & 92.31 \\
1000.0 & 0.0127 & 0.4936 & 2.51 & 97.49 \\
& \multicolumn{4}{c}{$T=457 \mathrm{~K}$} \\
0.1 & 1.0000 & 0.0000 & 100.00 & 0.00 \\
1.0 & 0.9996 & 0.0002 & 99.98 & 0.02 \\
10.0 & 0.9963 & 0.0019 & 99.81 & 0.19 \\
100.0 & 0.9646 & 0.0177 & 98.20 & 1.80 \\
1000.0 & 0.7564 & 0.1218 & 86.13 & 13.87 \\
\hline
\end{tabular}

${ }^{\mathrm{a}} n_{\mathrm{m}}$ and $n_{\mathrm{d}}$ are relative mole numbers of monomer and dimer, $x_{\mathrm{m}}$ and $x_{\mathrm{d}}$ are mole $\%$ of monomer and dimer, respectively. 